\title{
Progress and Visions in Future Neutron Imaging - with the Focus on Concrete - Moisture Interactions
}

\author{
E. H. Lehmann, A. Kaestner, and S. Hartmann \\ Villigen PSI, Switzerland \\ Corresponding author: E.H. Lehmann, e-mail: eberhard.lehmann@psi.ch, Tel. +41563102963
}

Neutron Imaging \& Activation Group, Spallation Neutron Source Dicision, Paul Scherrer Institut, CH-5232

\begin{abstract}
Since the beginning of neutron imaging in Switzerland in the 90ies of last century, where pictures were taken with film methods, intensive work was done to develop this technique towards a real research tool. Not only the building of new dedicated beam lines and the development of new detectors and investigation methods but also the full understanding of the neutron transport and the interaction of neutrons with the sample material delivered this progress. Now we are able to study the objects in three dimensions (tomography) and in short time (milli-seconds), we can select the most suitable energy range for the investigation and scale the field-of-view and the spatial resolution according the demands from tens of centimetres down to tens of micro-meters. At one and the same facility complementary images with neutrons and X-rays can be performed under identical imaging conditions. New imaging techniques which are phase based have been introduced and applied for practical purposes. The community dealing with concrete and other porous structural materials did already take profit from some of those new techniques and is invited to continue in the most efficient way. The future trend will be given by a new generation of neutron sources which deliver a pulse structure and enable a perfect energy resolution via the time-of-flight techniques. Dedicated beam lines for neutron imaging are on the way to be designed and installed at those new sources soon.
\end{abstract}

Keywords: neutron tomography, digital imaging, spatial resolution, phase contrast, pulsed neutron sources 


\section{State of the Art in Neutron Imaging}

\subsection{Neutron Imaging Facilities at PSI}

The method of neutron imaging has started about 50 years ago as "neutron radiography" where samples were inspected at beam lines in transmission mode with the help of X-ray films excited by the neutrons with the help of a neutron-to-gamma converter. It lasted until the middle of the 90ies of last century when new detection methods came into use which were mainly digital ones. In the course of the more efficient utilisation of the neutrons for imaging (30 minutes per image before, seconds and less now) a lot of new methodical developments took place and new application fields were identified. This gave reasons to build new and dedicated beam lines at several places round the world and to improve the old ones.

Paul Scherrer Institut is proud to host at least two beam lines for neutron imaging purposes, one with a thermal spectrum (NEUTRA), the other with a cold one (ICON). A third beam line is under construction and will be accessible partly for imaging purposes. All three facilities are aligned at the Swiss spallation neutron source SINQ which is driven by the $590 \mathrm{MeV}$ proton cyclotron. This source operates without any nuclear fuel and is comparable in its performance to a research reactor of about $15 \mathrm{MW}$ power.

\subsubsection{The Spallation Neutron Source SINQ}

SINQ is the national large scale facility for neutron research in Switzerland. Its physical principle is the split of heavy nuclei (lead) by the bombardment of high energy particles (protons) - spallation. About 10 fast neutrons are emitted per spallation act which are slowed down within the heavy water moderator to the thermal energy around $25 \mathrm{meV}$ and further down to about $3 \mathrm{meV}$ in the cold source, a volume filled with liquid deuterium kept stable on a temperature level of $25 \mathrm{~K}$.

Around the spallation source we can find more than 17 individual experimental facilities using the neutron beams, mainly devoted to neutron scattering. As mentioned above, three imaging beam lines cover three beam ports and provide high performance for different kinds of neutron imaging studies. They are built very flexibly in order to support as many different applications as possible from scientific and industrial users round the world. About 100 different projects are performed per year, most of them within the user proposal access.

\subsubsection{NEUTRA - Thermal Neutron Imaging Facility}

This facility was installed and introduced along to the SINQ setup until 1997 [1]. It consists of two experimental positions, one for large objects (up to $40 \mathrm{~cm}$ in diameter), and the other for higher resolution and for time series due to the delivered higher neutron beam intensity. Accordingly, two fixed camera based detection systems are installed which are flexible in the field-of-view in a certain range. At both positions, the use of a rotation table allows to perform tomography studies by taking projections when the object is rotated around its vertical (or horizontal) axis. Beside the thermal neutron beam, X-rays can be provided alternatively from an X-ray tube with up to $320 \mathrm{kV}$ high voltage.

\subsubsection{ICON - Cold Neutron Imaging Facility}

This beam line was set into operation in 2006 [2] in complement to NEUTRA in order to provide an alternative neutron spectrum for imaging purposes. Cold neutrons are usefully to enhance the contrast for most materials and to enable potentially a higher spatial resolution. For this purpose, a camera based detection system was built with an inherent pixel size of $13.5 \mu \mathrm{m}$ and optimal light transmission from the scintillator to the sensor [3]. This device is heavily in use for micro-tomography at a beam position at about $6.5 \mathrm{~m}$ from the cold source. Further downstream, at about $11 \mathrm{~m}$ a second detector position is installed for large objects with about $40 \mathrm{~cm}$ in diameter. A sample manipulator is able to carry objects with up to $500 \mathrm{~kg}$ with a precision in the positioning of $0.1 \mathrm{~mm}$. ICON is foreseen to provide an energy selective beam with $15 \%$ resolution which can be applied for dedicated investigations of crystalline structural materials (see below).

\subsubsection{BOA - Test Beam Line with Polarized Neutrons}

This beam line is aligned to the same cold source position as ICON. A suppression of shorter wavelength is provided by means of a curved bender in front of the beam exit. This bender enables the separation of one of the two spin states of the neutron and delivers a high degree of polarized neutrons. The future use of this beam line depends on the degree of sharing with other test applications. 


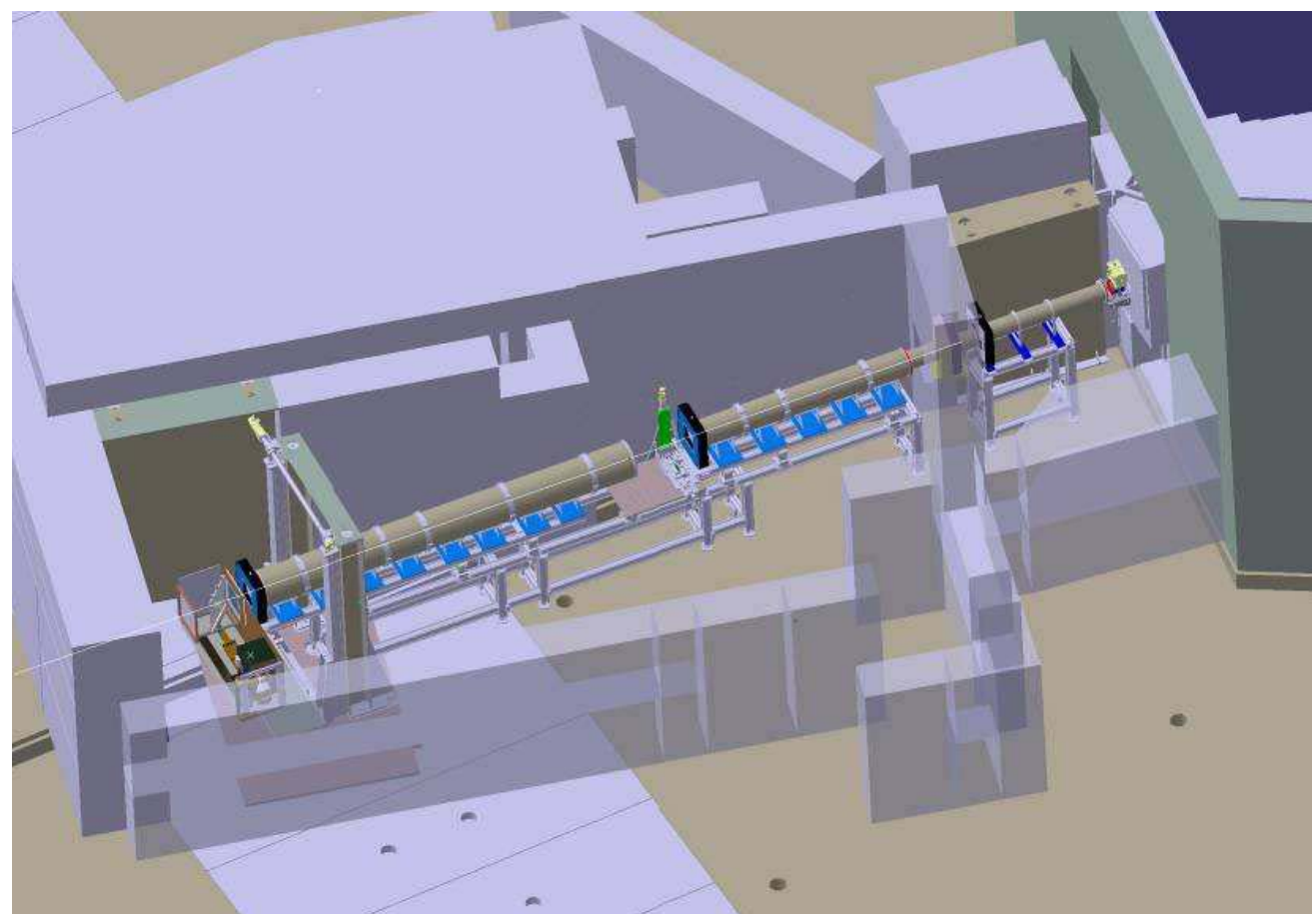

Figure 1: The neutron imaging facility ICON at beam port 52 of the spallation neutron source SINQ providing cold neutrons for different studies and industrial applications

\subsection{Advanced Neutron Imaging Tech- niques}

The major progress in neutron imaging started in the 90ies of last century with the development and implementation of new digital detection systems which provide many advantages compared to the traditional analogue methods and pushed also new physical approaches in neutron imaging. Although several such detection systems are now available, the dominating technique is the camera based one with a scintillation screen as neutron-to-light converter (see Fig. 2). It provides the highest flexibility in respect to the field-of-view and the spatial resolution while the signal-to-noise ration can be made superior to other techniques. The dynamic range and the linearity are very high.

The overview of the three available camera detection systems is given in Fig. 3, indicating the achievable field-of-view and the corresponding spatial resolution.

In addition, we have also available neutron sensitive imaging plates (replacing the old film techniques), amorphous Si flat panel detectors and pixel detectors for test purposes.

This flexibility in the detection system makes it possible to decide carefully from cases to case in the user program what is the most suitable imaging device for the particular application.

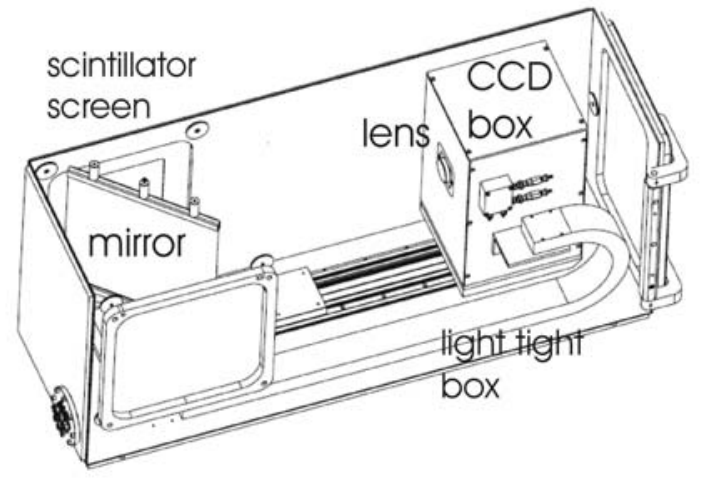

Figure 2: Layout of the detector box for neutron imaging using a cooled CCD looking onto a neutron sensitive scintillation screen

\subsubsection{Neutron Tomography}

This method enables to obtain the full 3-dimensional information of an object under investigation. A digital neutron area detector with at least 1000 pixels per direction is required which has to be stationary aligned in the beam behind the object. As shown with the example of a concrete sample in Fig. 4, tomography provides much more details compared to the radiography image, which is integrating all layers in beam direction. Virtual slices by means of software tools are possible in all directions at arbitrary positions. 
Detector options with CCDs

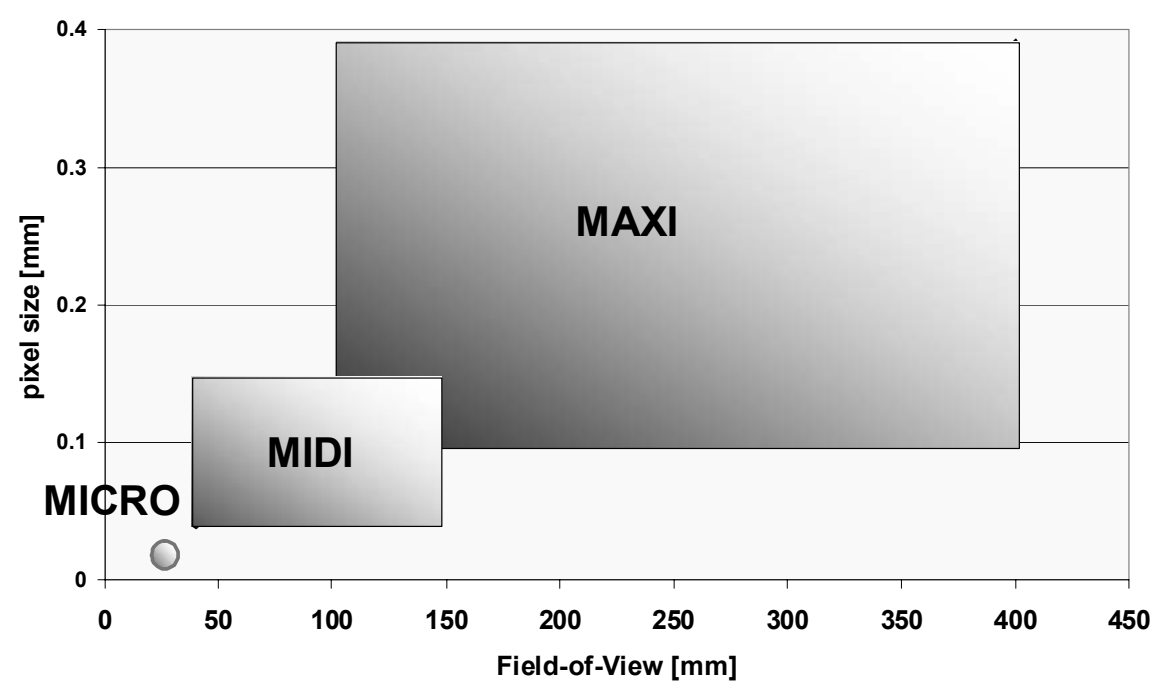

Figure 3: Working range of the different detection systems in respect to their geometrical alignment
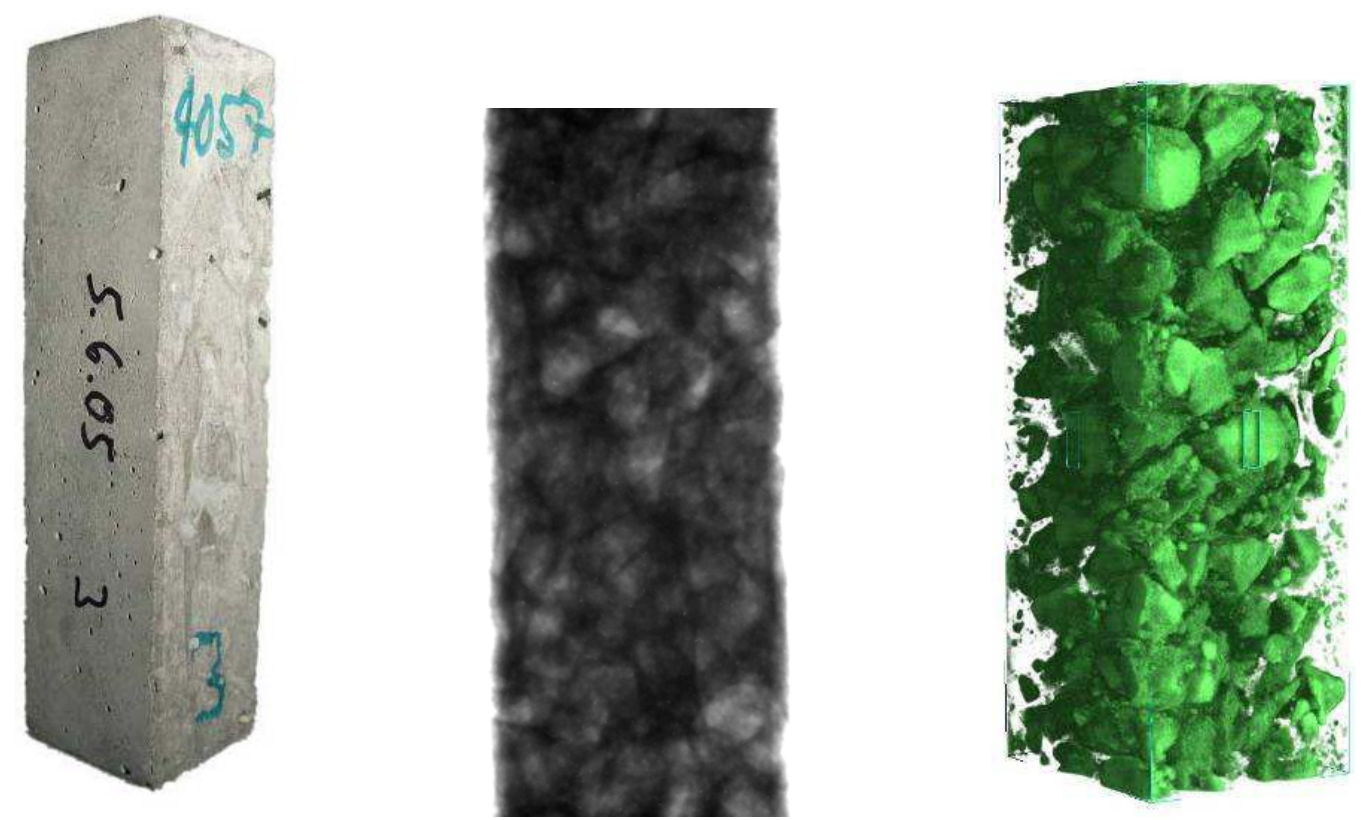

Figure 4: Investigation of the composite structure of a concrete sample. Left: c photographic view, centre: neutron transmission radiography, and right: neutron tomography

The price for a more informative data set is the longer acquisition time because the tomographic reconstruction of the third dimension by means of mathematical methods is based on at least 300 single projection which have to be taken while the object is rotated around its vertical (or horizontal) axis. The application of the reconstruction algorithm needs an advanced computer performance and takes some extra time in the minutes to hours range, depending on the size of the data set and the used algorithm.

Therefore, a single neutron transmission image can be obtained within seconds while the tomography results are available in time ranges of hours only. It is worth to decide if and how a tomography study is required for the particular problem to solve. 


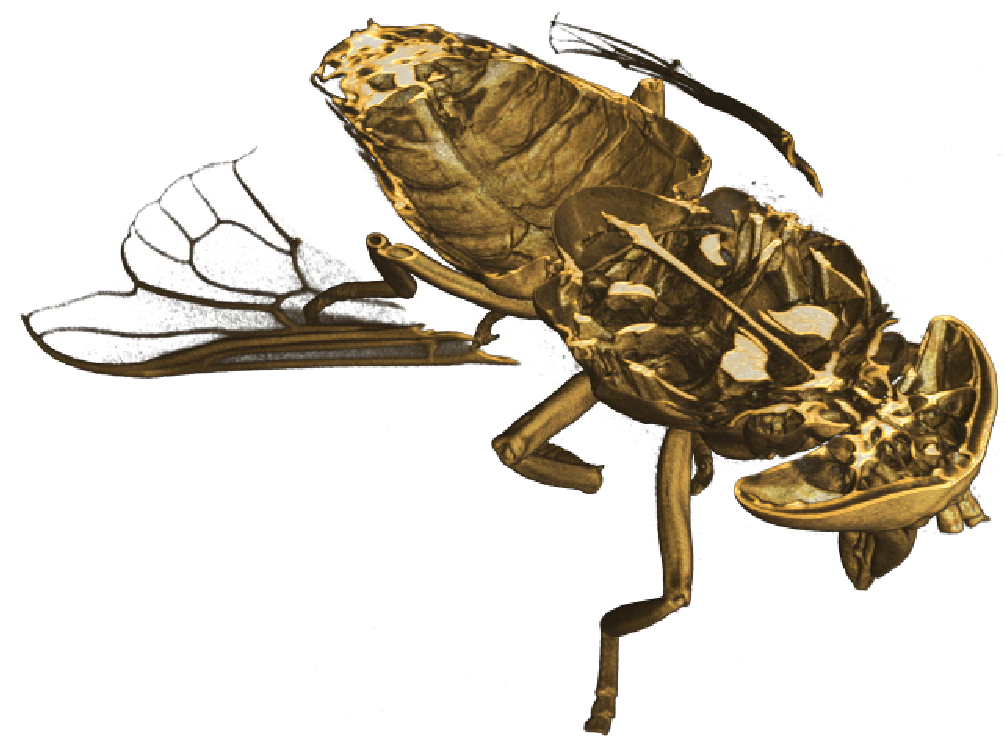

Figure 5: Example from the high resolution neutron tomography: horsefly; length scale about $12 \mathrm{~mm}$, voxel size $13.5 \mu \mathrm{m}$; the high contrast for organic materials can be exploited favourably in this case

Neutron tomography [4] is established at the two beam lines NEUTRA and ICON with the same performance as described by the data in Fig. 3. An example for the presently highest spatial resolution in our neutron tomography system is shown in Fig. 5, the lower half of a horsefly.

\subsubsection{Real-Time-Imaging}

The exposure time in neutron imaging can be reduced down to the range below one second by several means. On the one hand, the spatial resolution can be optimized when several pixels are combined (binning). On the other hand, the image quality can be sacrificed and the signal-to-noise ratio limited. Beside the real neutron exposure also the read-out time has to be taken into account in fast imaging.

Just recently, it was possible to image in "slow motion" a combustion motor in real operation with $8.000 \mathrm{rpm}$ in order to study the inner processes. While the structure is transparent for neutrons, lubricants and even fuel distributions become visible.

This single frame in Fig. 6 was obtained by triggering the detector in a short time frame of $50 \mu \mathrm{s}$ and stacking of images with the same positions of the engine over many operation cycles.

\subsubsection{Phase-contrast Imaging}

Beside the absorption contrast which is usually used in transmission neutron imaging there was found another interaction mechanism which is based on the wave properties of neutrons. In this model picture as wave, a phase shift takes place

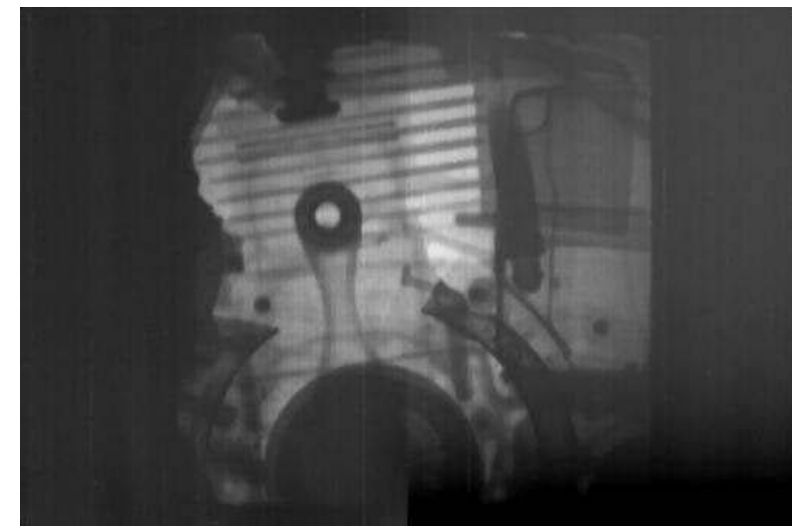

Figure 6: Stroboscopic image of a combustion engine obtained in real-time mode when the rotation speed was about $8.000 \mathrm{rpm}$

when neutrons interact with the sample. With the help of suitable experimental setups it becomes possible to extract explicitly this phase signal [5] or to use phase effects for the enhancement of edges near weakly visible materials. This refraction based phenomenon can be observed with the high resolving imaging systems, in particular when neutrons with a long wavelength $\lambda$ (cold neutrons) are involved. The neutron refraction index $\mathrm{n}$ can be described by the following relation:

$$
n=1-\delta=1-N \cdot \sigma_{\text {coherent }} \cdot \lambda^{2} / 2 \pi
$$

Here, the atomic density $\mathrm{N}$ of the material under investigation and its coherent scattering cross-section $\sigma$ have to be taken into account. The deviation from 1 is in the order of $10^{-5}$ only, but measurable. 

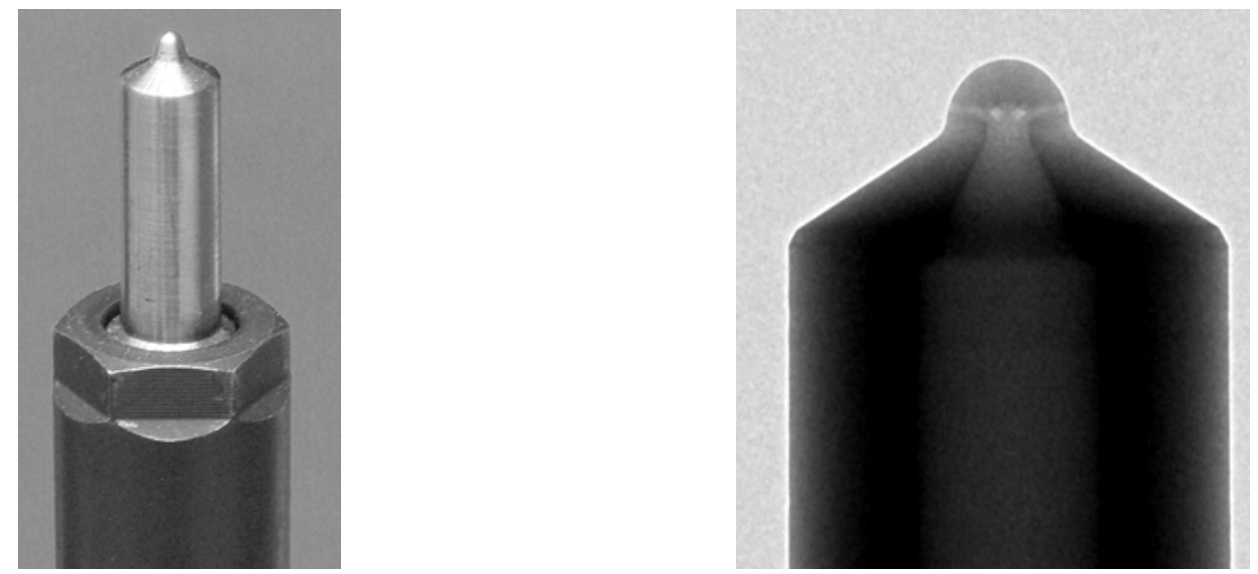

Figure 7: Edge enhancement effect; bright sky shine around the sample close to the edge by refraction in the steel injection nozzle of a Diesel engine due to the coherent scattering of cold neutrons; left a photo of the nozzle is shown

\subsubsection{Energy Selective Imaging}

Although most of the routine neutron imaging investigations have been performed with the full spectrum of the arriving neutrons (cold or thermal), a narrowing of the energy band provides some new scientific aspects. As crystalline structural material scatter neutrons at their lattice planes according to Bragg's law, a visualisation of crystallite orientations becomes possible if the most suitable neutron energy is selected. Furthermore, the quantification in transmission mode becomes much more precise if only a narrow energy band has to be considered.

Future imaging facilities at pulsed neutron sources, now under construction and consideration will enable a much higher degree of energy selection by using time-of-flight techniques.

\section{Applications}

The modern techniques in neutron imaging attract many users from the scientific and industrial community. Due to a high degree of flexibility, the imaging facilities at PSI are more than booked out during the 8 month operation time per year. The access is organized for scientific users within a proposal procedure with two calls per year. Paid industrial projects are invited in a more flexible regime by direct offers of beam time on request.

\subsection{Water Migration in Time and Space - Quantification}

Due to the high sensitivity of neutrons for the observation of water in porous media we performed many different studies with concrete, stones, wood and textiles as the base structural materials in respect to their uptake and transport of moisture.

However, not only the visualisation of water but also the quantification of its amount has a high importance. Unfortunately, high concentrations of hydrogen which is a strong diffuse scattering material limit the direct quantification of the water amount. Therefore, an image correction tool - QNI - was developed in order to extract the amount of secondary scattered neutrons. QNI [6] is based on Monte-Carlo simulations of the transmission process and has demonstrated its good performance within studies for soil physics and wood research [7] and materials physics [8].

QNI has been implemented mainly for thermal neutrons and hydrogen. Future activities at PSI will be concentrated to study the effects with cold neutrons. Furthermore, the scattering in the Bragg region will be taken into account explicitly. This is of high relevance for most of the crystalline structural materials $(\mathrm{Fe}, \mathrm{Cu}, \mathrm{Ni}, \mathrm{Al}, \mathrm{Pb}, \mathrm{Zr}, \ldots)$.

\subsection{Analysis of Structures}

The non-invasive study of the material composition and structure (geometry of the material distribution) is possible with neutrons either in direct transmission mode or with tomography methods for retaining the full volumetric information. Both techniques determine the quantitative information in form of a macroscopic cross-section $\Sigma$ when the exponential law of attenuation is applicable:

$$
\Sigma=\ln \left(\frac{I_{0}}{I}\right) / d
$$



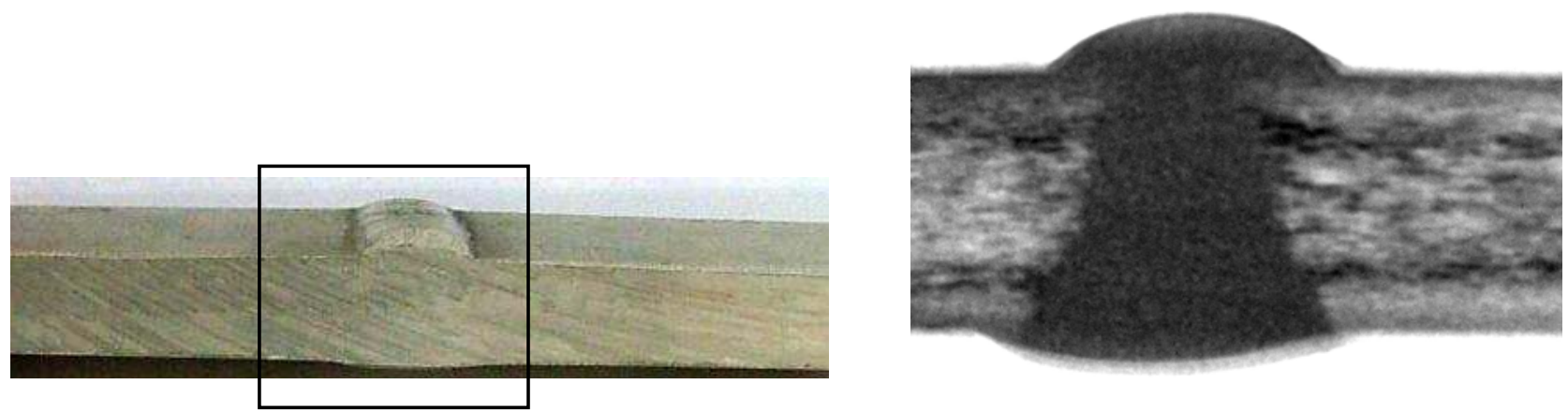

Figure 8: Results of energy selective imaging (made at $\lambda=3 \AA$ ) of the weld between two rolled $\mathrm{Al}$ plates; the micro-structure obtained from the manufacturing process (grains, grain size orientation) can directly be analysed while the weld itself shows a more homogenous structure
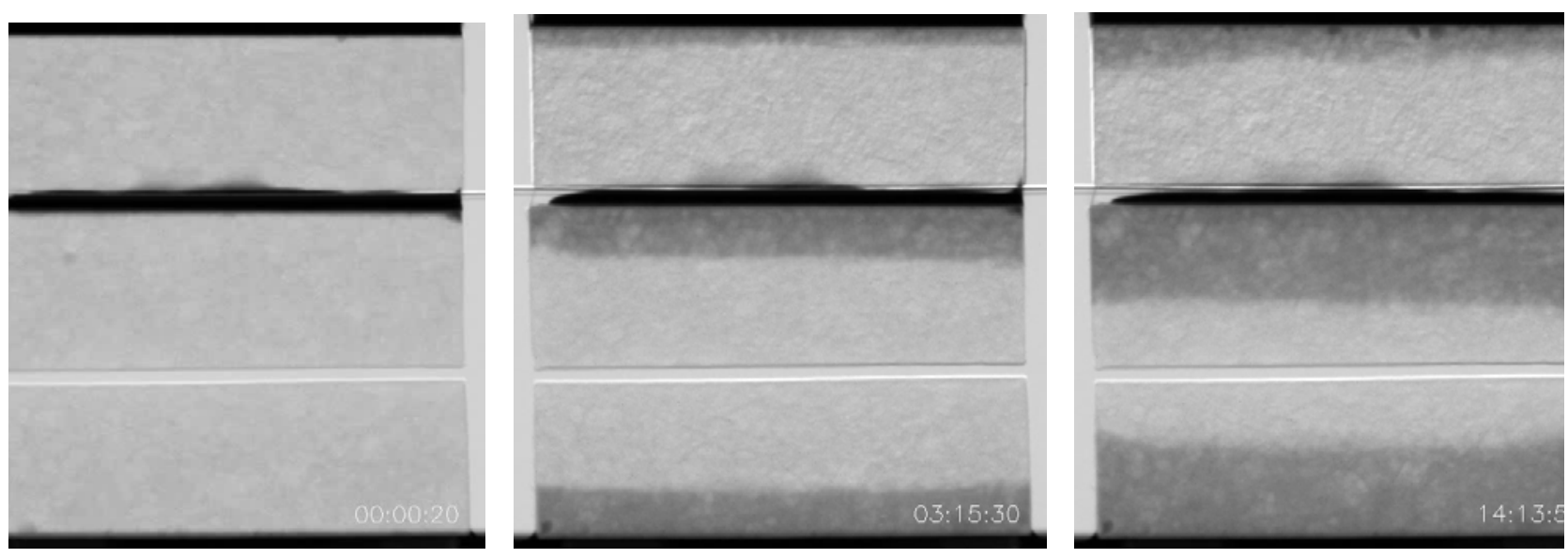

Figure 9: Example for image referencing: Three concrete samples were kept stable in their position while silane was applied for impregnation purposes. In this manner, the structure of the concrete nearly vanished while the silane amount provides a strong grey contrast. Data are taken from [9]

Three parameters need to be known: the initial beam intensity $\mathrm{I}_{0}$, the intensity behind the object I and their thickness $\mathrm{d}$. The macroscopic cross-section relates to the material density $\rho$ in the following manner:

$$
\Sigma=\frac{\rho \cdot L}{M} \cdot \sigma
$$

The microscopic cross-section $\sigma$ describes the probability of the interaction of neutrons of a given energy with the sample material (atomic weight $\mathrm{M}$ ) and is known in first order and tabulated. The sensitivity to detect and to investigate specific materials in high sensitivity depends clearly on the values of $\sigma$. Prominent agents for a high contrast are hydrogen and the strong absorbers B, Li, Cd or Gd. They can be detected in small amounts within bulk samples of even heavy elements.
In digital neutron imaging, each pixel (or voxel in tomography) is attributed to a $\Sigma$ value. It depends on the contrast in neighbouring pixels how effective it is to distinguish them and to enable it to find defects, structural changes or material edges. Practical applications in this respect are the studies of adhesive layers, soldering connections, pyrotechnical devices, the water accumulation in electro-chemical fuel cells and the gas production in Li-ion batteries.

\subsection{Defects and Material Treatment}

In respect to defects in materials some advanced methods become interesting. If structural defects cannot be found easily due to the missing image contrast, phase contrast information might be used instead. As the wave-shift at the position of the defect is high enough, a visualisation might be enabled in some cases better. This method has to be improved more by practical experience. 
Energy selective imaging is another useful tool for material research. It has been demonstrated e.g. for rolled $\mathrm{Al}$ plates that the texture in the different layers becomes directly visible if neutron energy bands are chosen near the Bragg edges of $\mathrm{Al}$ (see Fig. 8). In the same way, we can study material changes if load is applied to a sample and stress is induced.

\section{Studies for Concrete Knowledge Improvement}

Digital detectors which can be aligned stationary fixed in the beam are used for pixel-wise referencing in time series. In this manner, the sensitivity for the detection and quantification of the moisture changes is increased dramatically. With this option, neutron imaging can be considered to be the most sensitive method for moisture determination with best resolution in time and space.

In particular, when the matrix of a setup (stone, wood, textile, ...) remains not stable at its position, but migrates during the water uptake and loss, we are using the so-called X-TRA option at the NEUTRA beam line for referencing with an additional image data set taken under identical beam conditions. This option might overcome the problem of the sample volume change as the X-TRA images are not or only little affected by the water content.

The high flexibility for the sample size (in respect to the filed of view of the detector) and the inherent spatial resolution (as shown in Fig. 3) are important for the solution of different kinds of problems in building material technology and treatments. Prominent requests are the stability of water repellent layers, the water migration near cracks and the salt ingress into the concrete matrix.

We see a further potential in the use of energy selective methods where the contrast for the different process components can be enhanced and the detection probability can be improved. It will be a process of further test investigations to find out the best conditions for the particular study. Further potential users are invited to join us for these investigations.

\section{Conclusions}

Neutron imaging has been established as a valuable tool for non-invasive and non-destructive studies in a number of laboratories around the world. Different new techniques are still under development and will give further impact to the user com- munity. Major challenges are the improvement of the spatial resolution, the implementation of phase-contrast methods as research tools and the use of energy-selective methods as a link to the neutron scattering techniques for the analysis of crystalline solid state materials.

\section{References}

1. E. Lehmann and H. Pleinert, The new neutron radiography station at the spallation source SINQ, INSIGHT Vol. 40, No. 3, (1998)

2. $\quad$ A.P. Kaestner et al., The ICON beamline - $A$ facility for cold neutron imaging at SINQ, Nucl. Instr. \& Meth. A, Vil. 659, Issue 1, pp. 387-393

3. E.H. Lehmann et al., The micro-setup for neutron imaging: A major step forward to improve the spatial resolution, Nucl. Instr. \& Meth. in Phys. Res. A, Vol. 576, Issue 2-3, pp. 389-396 (2007)

4. P. Vontobel, E.H. Lehmann, R. Hassanein, G. Frei, Neutron tomography: Method and applications, Physica B 385-386, 475-480 (2006)

5. F. Pfeiffer, C. Grünzweig, O. Bunk, G. Frei, E. Lehmann, C. David, Neutron Phase Imaging and Tomography, Phys. Rev. Lett. 96, 215505 (2006)

6. R. Hassanein, Correction methods for the quantitative evaluation of thermal neutron tomography, Dissertation 16809, ETH Zurich, Zurich (2006)

7. E. Lehmann, P. Vontobel, P. Scherrer, P. Niemz, Application of neutron radiography as method in the analysis of wood, Holz Roh- Werkst 59(6):463-471 (2001a)

8. P. Zhang, F. H. Wittmann, T. Zhao, E. H. Lehmann, Neutron imaging of water penetration into cracked steel reinforced concrete, Physica B: Condensed Matter, 405(7), 1866-1871 (2010)

9. A. Marlow, Untersuchungen zum Silantransport in der Betonrandzone mittels Neutronenradiographie, Diploma Work, Fachhochschule Karlsruhe (2005) 


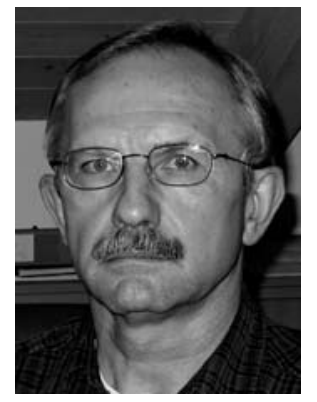

\section{Dr. Eberhard Helmar Lehmann}

1970-1974: study in physics at the University of Leipzig (Germany) - diploma as "Dipl.-Physicist", Topic: "Molecular dynamic calculations of proteins"; 1983: Dr. rer. nat. at the German Academy of Science, Berlin; Topic: "Cross-section data of construction materials for the fast breeder reactor by reactivity measurements".

Present Position: Group Leader "Neutron Imaging \& Activation" of the Department Spallation Neutron Source, Paul Scherrer Institut, Switzerland.

Field of Specialisation: Neutron Physics for Research Reactors and Spallation Sources, Applications of Neutrons in Science and Industry; Neutron Imaging, Irradiation Technology. Experience: 1976-1990: research in reactor physics for fast breeders, experimental work at different reactor stations in several countries, calculations of reactor parameters with different reactor codes; 1991-1995: reactor physicist at the research reactor SAPHIR, responsibility for core design and applications; since 1995: responsibility for non-diffractive applications and spallation neutron physics of the spallation neutron source SINQ at the Paul Scherrer Institute and for neutron utilisation around the source.

E-mail: eberhard.lehmann@psi.ch

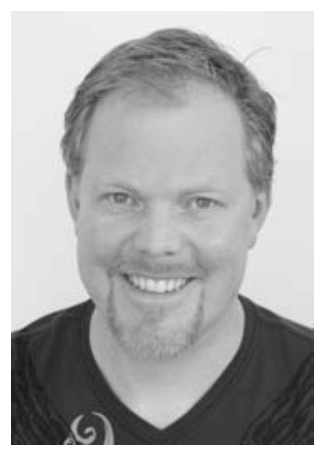

Dr. Anders Kaestner was born in Helsingborg, Sweden, in 1971. He is a beamline scientist at the cold neutron imaging beamline (ICON) at Paul Scherrer Institut, Switzerland. He studied computer systems engineering with specialization to mechatronics at Halmstad University, Sweden, and received a MSc. in 1997. He started working with imaging using microwaves during his masters thesis. The work with imaging and image processing was continued with a PhD in Signal Processing at Chalmers Institute of Technology, Gothenburg, Sweden, in 2002. The work with image processing continued as a postdoc working with image processing tasks for soil science in the soil physics group at the institute for terrestrial ecology, Swiss Federal Institute of Technology in Zürich, Switzerland. Special fields of interest are computed tomography and image enhancement. He is currently a board member of the international society for neutron radiology.

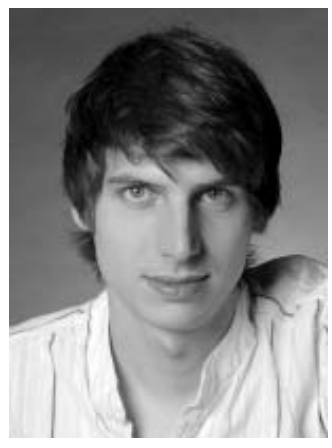

Stefan Hartmann has a mechanical engineering degree from UAS in Windisch. Since 2004 he works with neutron imaging at the spallation neutron source SINQ as well as with the development and improvement of its neutron imaging beam line process control systems.

Received January 5, 2012 
